Boyne, A. W., EAdIe, J. M. \& RaItT, K. (1957). J. gen. Microbiol. 17, 414-423

\title{
The Development and Testing of a Method of Counting Rumen Ciliate Protozoa
}

\author{
By A. W. BOYNE, J. MARGARET EADIE AND KATHLEEN RAITT \\ The Rowett Research Institute, Bucksburn, Aberdeenshire
}

SUMMARY: The limitations of methods previously used for counting rumen ciliates, and the value of developing a method of known accuracy, are discussed. Two counting-chambers, one based on the Sedgwick-Rafter cell, the other on the MacMaster eelworm-counting cell, were constructed and tested. A MacMaster-type cell was adopted. The preparations of suitable suspensions for counting organisms from washed suspensions and from rumen contents are described. The counting technique adopted was found to be reliable; its accuracy depends upon the number of organisms counted. In one sheep on a constant diet, day-to-day differences in rumen ciliate population were far greater than the differences found throughout the rumen at any one time, or the variations due to technique.

Many workers studying rumen protozoal fauna have given counts of ciliate protozoa in their results. Their object has most frequently been to compare ciliate populations after changes of diet in an attempt to correlate ciliate population with digestion in the animal (Ferber, 1928; Ferber \& Winogradawa-Federowa, 1929; Mowry \& Becker, 1930); the work of van der Wath \& Myburgh (1941), Weller \& Gray (1954), Moir (1951) and Williams \& Moir (1951) had much the same objective. Hungate (1942) in the investigation of the division rate of his cultures of Eudiplodinium spp., illustrated the use of a counting method for in vitro studies. Gutierrez (1955) made biochemical examinations of the holotrich protozoa in vitro, and used counts made from these experiments and from whole rumen liquor in order to determine the contribution of the holotrichs to volatile fatty acid production in vivo. However, despite the significance frequently attached to the results very little attention seems to have been given to the development of a counting method of known reliability and there has been little consistency either in the methods used or in the results obtained. Oxford (1955) enumerated some of the difficulties involved in making accurate rumen ciliate counts, but also stressed the importance of being able to assess the total and differential population (e.g. holotrichs and various kinds of oligotrichs) in order to relate the contributions of particular ciliates to ruminant digestion. Adam (1951), after describing her method for counting horse intestinal ciliate protozoa, pointed out that she was unable to compare these results with total rumen ciliate counts because no really reliable results were available. Her most obvious criticism of the total rumen ciliate counts available was that there was no means of assessing how far the small rumen samples used were truly representative. It is clear that until a reliable method is found to determine with a known degree of accuracy the concentration of protozoa in a given rumen sample, no conclusions can be drawn as to the value of that sample as a 
representative of the rumen as a whole. Although it might eventually be found that in routine counts certain experimental refinements may be unnecessary in the light of differences between sheep on the same ration, it is first of all essential to know how much of the total variability encountered on any occasion is due to the method used. The present paper describes the development of a method, based on that of Adam (1951), by which counts of rumen ciliates both in vitro and from total rumen liquor, may be obtained, with known accuracy.

\section{METHODS}

Rumen ciliate protozoa have greater variation in size than protozoa from many other sources. Many counting methods, e.g. nephelometry, depend on the organisms being of uniform size. Previous workers have used counting chambers of standard design and most work on rumen ciliate counts has been done with haemocytometers (Ferber, 1928; Johnson, Hamilton, Robinson \& Garey, 1944; Weller \& Gray, 1954; Moir, 1951). These blood-cell counting chambers (e.g. Fuchs Rosenthal used by Ferber) are usually $\mathbf{0 . 2} \mathbf{~ m m}$. deep and, though accurate when counting blood corpuscles of $c .7 \mu$. diameter, would hardly be effective with organisms which may be as long as $200 \mu$. Although most of the rumen ciliates are small ( $<60 \mu$. long), if a true total and differential count is to be obtained another type of counting chamber is required.

Adam (1951, 1953) developed a method for counting horse intestinal ciliates, which also show a great variation in size, using the Sedgwick-Rafter cell previously found effective by Hall, Johnson \& Loefer (1935) for counting pure suspensions of ciliates, and by Gilbert (1942) for counting phytoplankton varying in size from single to colonial forms. The Sedgwick-Rafter cell has the added advantage that the capacity is larger than that of a haemocytometer and it is therefore less subject to sampling error. It is noteworthy that Moir \& Somers (1956) when counting rumen ciliates of sheep have recently turned from the use of a haemocytometer (Williams \& Moir, 1951) to a modified form of Adam's technique, but no details are given.

Preparation of a Sedgwick-Rafter type of counting cell similar to that of Adam. A counting chamber of exactly $1 \mathrm{ml}$. capacity, based on that used by Adam (1951), was prepared with a 3 in. $\times 1.5$ in. microscope slide as base. Strips of normal microscope slides exactly $1 \mathrm{~mm}$. in thickness were firmly attached to it to give a chamber exactly $1 \mathrm{ml}$. in volume. A commercial cement 'Coaguline' (Kay Brothers Ltd., Stockport) was used to fix the strips, the layer required being so thin that it did not affect the dimensions. A large coverslip was carefully cut to cover the $20 \times 50 \mathrm{~mm}$. oblong cavity and to rest on the platform formed by the cut sections of slides.

The function of the Sedgwick-Rafter cell as used by Adam was to obtain a layer of suspended organisms $1 \mathrm{~mm}$. deep, to allow them to sink to the base of the cell and then to be able to count random $1 \mathrm{~mm}^{2}$ areas by the use of an eyepiece with an accurately marked area of $1 \mathrm{~mm} .{ }^{2}$ when projected. In this way the organisms originally in $1 \mathrm{~mm} .{ }^{3}$ of the fluid could be counted.

Preparation of a cell based on that used by Adam and the MacMaster eelworm 
type of counting cell. The second counting chamber prepared was like the MacMaster cell in design and was composed simply of a normal microscope slide firmly fixed $1 \mathrm{~mm}$. above a large $3 \mathrm{in} . \times 1.5$ in. slide, leaving a space into which the suspension was drawn and held in place by surface tension. Two platforms made from strips of microscope slides exactly $1 \mathrm{~mm}$. thick were fixed $20 \mathrm{~mm}$. apart on the base slide and the upper slide firmly attached to these. As before, 'Coaguline' was used as adhesive; this proved extremely firm and was unaffected by glycerol or frequent washing. A glass-pencil mark along the length of the upper slide allowed a regular check that no sagging had occurred. As microscope slides are optically level the only accurate measurement required was the $1 \mathrm{~mm}$. thickness of the side platforms.

Advantages and disadvantages of the cells and the final technique of counting adopted. Note was taken of the warnings of Gilbert (1942) and of Adam (1951) that care is required to fill the slide with a fixed volume of fluid suspension; it was never possible to fill the Sedgwick-Rafter cell with exactly $1 \mathrm{ml}$. of suspension and place the coverslip in position without the formation of an air bubble. This was caused by the escape of fluid between the coverglass and the platform. The bubble could be eliminated if slightly more than $1 \mathrm{ml}$. of suspension was used, but as it was noted that only fluid escaped, there was in effect a certain straining action and the organisms tended to be more concentrated round the edge of the cell. A MacMaster type of cell was therefore constructed and counts made with it were tested statistically.

The MacMaster type of cell could be filled with just over $1 \mathrm{ml}$. of suspension from a pipette held in one position at the edge and, provided the suspension was suitable (see later), it was possible to avoid air bubbles at the enclosed edge and to obtain what appeared to be an even suspension of organisms. The ciliates were denser than the suspending fluid, and after about $5 \mathrm{~min}$. had sunk to the base of the cell where they could be counted. A square micrometer eyepiece scale was calibrated to project an area of exactly $1 \mathrm{~mm} .^{2}$ on to the lower slide. The magnification used was limited by the necessity of having this $1 \mathrm{~mm}$. square completely within the field of vision. This was best achieved with a $\times 6$ eyepiece in the straight monocular body of an M-2000 microscope (Cooke, Troughton and Simms, York) using a $\frac{2}{3}$ in. achromatic objective. This gave a magnification of approximately $\times 60$ with which the organisms could be distinguished even when obtained from whole rumen liquor. The vernier scale of the microscope stage very conveniently gave fixed positions on the slide: nineteen positions lengthwise and eleven from top to bottom with a slight space between each field. A table of random numbers was used to obtain random positions on the slide.

It was difficult to prepare suspensions of organisms from which samples could be removed to fill the counting chamber. One possible error which has been noted but not adequately compensated by previous workers is due to the rapid sedimentation of some of the rumen ciliates. The object was therefore to obtain a suspension of organisms fixed in a manner which left them recognizable and suspended in a fluid in which the viscosity greatly decreased the sedimentation rate but nevertheless allowed the organisms to settle in a few 
minutes on the base of the counting chamber. Ferber (1928), whose counts are the most generally quoted, suspended the organisms in water which, besides being non-viscous, rapidly causes bursting of the holotrichs. Weller \& Gray (1954) used formalin, and Moir (1951) an iodine solution. Hungate (1942) and Gutierrez (1955) used suspensions in rumen fluid, and although thorough mixing was carried out Gutierrez made serial dilutions from the suspensions, and samples as small as $\mathbf{0} \cdot 1 \mathrm{ml}$. were removed. As certain rumen ciliates settle even in the course of emptying a pipette, the chances of error are very real, so that the effect of consistency of rumen liquor on the protozoa population, mentioned by Mangold (1929), may be real, but may equally well be due to bias in sampling because of the effect of consistency on the rate of settling.

Preparation of washed suspensions of protozoa. Suspensions consisting largely of holotrich organisms separated by the method of Heald, Oxford \& Sugden (1952) were used in testing various suspending media. These organisms when filled with storage material during the separation process were the most dense likely to be encountered. A series of suspensions in glycerol+buffer were tested (Adam, 1951). It was not found necessary to use a histological fixative since the glycerol rapidly killed the organisms and though it caused some distortion at first, within $5 \mathrm{~min}$. the organisms regained their original shape. It had the added advantage of clearing the ciliates so that even well-filled holotrich organisms were clearly distinguished by the position of the mouth, and no stain was necessary. An approximately $50 \%(\mathrm{v} / \mathrm{v})$ mixture of glycerol and buffer proved the most effective.

Preparation of suspensions of protozoa from rumen liquor. It was necessary to separate the protozoa from the debris in such a way as to avoid loss or death of protozoa and at the same time to retain only particles of debris of comparatively small size. This is particularly important because in the large counting chamber, all the material in $1 \mathrm{~mm} .^{3}$ is accumulated on an area of $1 \mathrm{~mm} .^{2}$ and pieces of debris which might mask protozoa must therefore be avoided. Adam (1951) appears to be the only worker who has seriously attempted to separate the organisms from debris.

A series of straining materials and methods was tested including muslin (as used by Adam), bolting silk, and nylon, but eventually metal gauze soldered on to a cylindrical tin in place of the base was found most effective. Sieves of mesh 40,80 and 120 were used in succession as described below. It was found essential to avoid copper in the sieve as the holotrichs were rapidly killed and disintegrated when copper, brass or monel metal gauze was employed. Instead, stainless steel gauze of woven wire cloth I.s.w.G. 41 (Baird and Tatlock, Chadwell Heath, Essex) was obtained and this was non-toxic.

The sample of rumen contents was passed through the coarse sieve into a 2 1. beaker containing some buffer ( $\mathrm{NaCl}, 0.5 \%$; anhydrous sodium acetate, $0.13 \% ; \mathrm{KH}_{2} \mathrm{PO}_{4}, 0.03 \% ; \mathrm{K}_{2} \mathrm{HPO}_{4}, 0.10 \% ; \mathrm{MgSO}_{4} .7 \mathrm{H}_{2} \mathrm{O}, 0.01 \%$; adjusted to $\mathrm{pH} 6.9$ to $7 \cdot 2$ ) and both sieve and buffer were kept in motion. The filtrate was poured into a second beaker and the sieve was further washed in fresh 
buffer several times until the washings were clear. The total filtrate was then put through the second sieve which was similarly washed and finally the process was repeated with the third sieve. Tests showed that movement of the fluid during washing and several washings of each sieve were essential to free the debris of ciliates. Numerous examinations of the residue in the sieve showed that practically all the organisms were washed through when the above method was used. This applied to the rumen contents of sheep on diets of hay, grass or concentrates. When $100 \mathrm{ml}$. rumen liquor samples were used it was found necessary to put the sample through the first sieve in two $50 \mathrm{ml}$. quantities. All the sieving was carried out in vessels standing in a $38^{\circ}$ water bath.

After sieving, the organisms from the original sample were all present in a large volume of buffer, $c .2$ l. for a $100 \mathrm{ml}$. sample. The organisms were then reconcentrated by centrifugation. The volume was first spun at $630 \mathrm{~g}$ for $10 \mathrm{~min}$., the deposits washed out with buffer and then pooled into one centrifuge pot and spun for $5 \mathrm{~min}$. at the same speed. The supernatant fluid was then free of protozoa when the sample was originally from a sheep fed hay and concentrates, but for different diets the speed and time of centrifugation might have to be varied: e.g. the time prolonged slightly for rumen liquor from a grass-fed animal. Excessive speed bursts the holotrichs and must be avoided.

Finally all the protozoa from the original sample were gathered into one centrifuge pot and were washed into the container from which samples were to be taken. This suspension was then made up to the desired volume and diluted with one volume of glycerol. The organisms were left for $15 \mathrm{~min}$. to recover shape. Further dilutions to give a suitable concentration for counting were made from this suspension, using a mixture of $50 \%(\mathrm{v} / \mathrm{v})$ glycerol in buffer solution.

Sampling of rumen contents and of protozoal suspensions. Important stages in the counting technique were the original removal of a rumen liquor sample from the sheep and the removal of samples to fill the counting chamber. All suspensions were made up in glycerol + buffer mixture in boiling tubes. The protozoa do not cling to the smooth sides of these tubes. A suspension was mixed by inverting the tube eight times while holding a hand over its mouth for the whole period of mixing so that any suspension removed would not affect the final concentration. After this mixing, the $1 \mathrm{ml}$. samples, to be diluted if necessary, were removed by pipette. The tips of the pipettes were ground down to be $2 \mathrm{~mm}$. in internal diameter; this is necessary to allow free passage of large and small organisms (Adam, 1951). The tip of the pipette was placed centrally in the tube before the sample was removed to decrease to a minimum error due to sedimentation. The viscosity of the suspensions was such that it took $5 \mathrm{~min}$. for starch-filled holotrichs to settle out.

The samples from the rumen were drawn by suction through a glass tube inserted into the fistula. The exact volume to be used in the count was drawn off so that no sampling from rumen contents in vitro was required, nor was any further sampling necessary until the glycerol was added. Rumen samples 
from the bottom or middle of the rumen were obtained by maintaining pressure on the upper end of the tube until it had been inserted into the rumen to the correct level.

\section{RESULTS}

\section{Test of cell with washed suspensions of holotrichs}

Experiment a. It had been shown by Student (1907) and confirmed by many subsequent workers that, provided no overcrowding occurs, there is a Poisson distribution of particles over the area of a haemocytometer. The similarity in principle of our counting slide to the haemocytometer led us to expect a similar result. To test this two fillings of the slide were made with mixed suspensions of Isotricha and Dasytricha spp. obtained from the rumen of a sheep fed hay and concentrates. The area of the slide was divided into 209 fields of $1 \mathrm{~mm} .{ }^{2}$ by using the vernier scale of the microscope. Each field might contain at least 50 of the Isotrichs or some hundreds of the Dasytrichs and the concentrations of the suspensions were such as to avoid any possibility of overcrowding. The results of this experiment are given in Table 1. Apart from

Table 1. Test of the counting method using two similar washed suspensions of rumen holotrich protozoa

Two samples of each suspension were counted.

\begin{tabular}{|c|c|c|c|c|c|}
\hline & \multirow{2}{*}{$\begin{array}{c}\text { Degrees } \\
\text { of } \\
\text { freedom }\end{array}$} & \multicolumn{2}{|c|}{ Suspension A } & \multicolumn{2}{|c|}{ Suspension B } \\
\hline & & $\begin{array}{l}\text { Isotricha } \\
\text { spp. }\end{array}$ & $\begin{array}{l}\text { Dasytricha } \\
\text { spp. }\end{array}$ & $\begin{array}{l}\text { Isotricha } \\
\text { spp. }\end{array}$ & $\begin{array}{l}\text { Dasytricha } \\
\text { spp. }\end{array}$ \\
\hline Mean count $/ \mathrm{mm}^{2}{ }^{2}$ field & - & $1 \cdot 0$ & $5 \cdot 3$ & 1.7 & $1 \cdot 4$ \\
\hline $\begin{array}{l}\text { Residual mean square of } \\
\text { transformed counts }\end{array}$ & 180 & $0 \cdot 20$ & $\mathbf{0} \cdot 30$ & 0.27 & $0 \cdot 28$ \\
\hline $\begin{array}{l}\text { Mean square attributable } \\
\text { to changes along the slide }\end{array}$ & 18 & $0 \cdot 23$ & $0 \cdot 31$ & $0 \cdot 37$ & $\mathbf{0 \cdot 2 4}$ \\
\hline $\begin{array}{l}\text { Mean square attributable } \\
\text { to changes across the slide }\end{array}$ & 10 & $0 \cdot 26$ & $\mathbf{0 \cdot 3 9}$ & 0.41 & $0 \cdot 14$ \\
\hline
\end{tabular}

the concentration of Dasytricha spp. from suspension $\mathbf{A}$, the means were rather small, and so, following Anscombe (1948), (count +0.375) $)^{\frac{1}{2}}$ was analysed for those three sets of counts. For the fourth, (count) $)^{\frac{1}{2}}$ was examined. Table 1 shows that the residual mean square was near the theoretical value of $0 \cdot 25$, and that the variability due to changes along or across the slide was not significantly greater. The data were therefore in accord with a Poisson distribution of the organisms over the slide.

It was thus possible to estimate, for a given average size of count, the number of fields which it is necessary to sample in order to estimate the concentration of organisms with some specified accuracy. The results for some representative accuracies are given in Fig. 1. These may be interpreted as follows. Suppose the average concentration is about $20 /$ field, and we wish to estimate the concentration to within $5 \%$. From the $5 \%$ curve we can see that if we count 80 fields the true mean will lie within $\pm 5 \%$ of the sample mean, c. 95 times 
out of every 100. From the nature of the distribution there will be some asymmetry, but the figure remains a useful guide to the sampling intensity required.

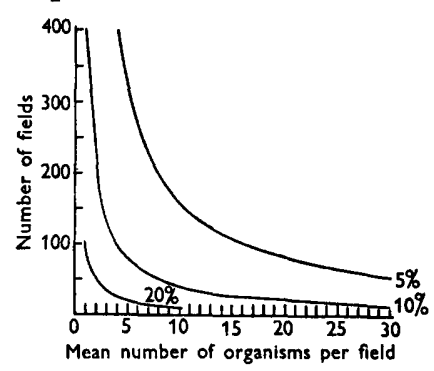

Fig. 1

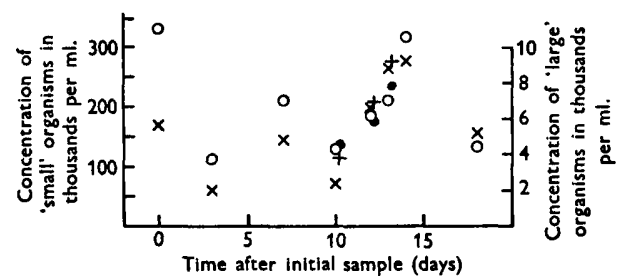

Fig. 2

Fig. 1. The number of fields which it is necessary to count for different concentrations of organisms so that the $95 \%$ confidence limits of the true mean should be within approximately $5 \%, 10 \%$, or $20 \%$ of the sample mean.

Fig. 2. Concentration of 'large' and 'small' organisms in rumen contents of a sheep sampled on different days.

\section{Test of the reliability of the slide counts as representatives of the volume sampled}

Experiment $b$ was carried out to see how well the sample drawn from a slide represented the material of its origin. Ideally, one would have a volume of a known concentration of holotrich organisms and sample this several times and examine the counts for bias and variability. It was not practicable to do this, nor was it considered at all likely that any bias would arise, so that this test was restricted to examining the differences which occurred among slides from one suspension. Experiments were carried out which exposed sources of differences between slides, until eventually the technique of mixing described above was evolved. Three experiments were then made to test the sampling method. In each of these washed holotrich organisms were prepared, and suspensions in glycerol + buffer solution were made. A sufficiently large volume was made up to render unimportant the effect of finite sampling fractions (Quenouille, 1950). The slide was filled seven times from each suspension and the organisms counted in thirty fields chosen at random from each slide. Table 2 lists the principal results of these experiments, in which (count $+0 \cdot 375)^{\frac{1}{1}}$ was analysed. The listed mean squares of the transformed count confirmed the result of Expt. $a$ in showing good agreement with 0.25. For each solution the mean counts on each side agreed well with one another; an analysis of variance of the transformed counts showed that slide differences were not significant. The appropriate mean squares are given in the last two rows of Table 2.

\section{$A$ test of the method applied to total rumen contents}

A Cheviot ewe fed a constant daily ration of $450 \mathrm{~g}$. hay and $450 \mathrm{~g}$. concentrates was used throughout Expt. c. At 10 a.m. on each of 8 days (over a 19-day period) one $100 \mathrm{ml}$. sample was withdrawn from the top, and another from the bottom, of the rumen. At 3 p.m. on 3 of these days two $100 \mathrm{ml}$. 
samples were taken from the middle of the rumen. The ciliates were prepared for counting as described above, and as far as possible each stage in the preparation was carried out concurrently for each pair of samples. The organisms from each $100 \mathrm{ml}$. sample were finally concentrated in $60 \mathrm{ml}$. of glycerol + buffer solution. To facilitate counting the 'large organisms' (large oligotrichs and Isotricha species) a 1/5 dilution was used, and for 'small organisms' (Dasytricha, Diplodinium and Entodinium species) the solution was diluted 1/40. Two slides were prepared of each suspension, and the large organisms were counted in thirty fields on each slide of the more concentrated solution, the small organisms in twenty fields on each of their slides. When the within-slide variability of the transformed count was analysed, the mean squares ranged from $0 \cdot 16$ to $0 \cdot 38$, with an average value of $0 \cdot 29$, so that the protozoa were distributed over each slide in a random manner.

Table 2. Test of the counting method using three washed suspensions of rumen holotrich protozoa

The counting chamber was filled seven times from each suspension and the organisms each time counted in $\mathbf{3 0}$ fields chosen at random.

\begin{tabular}{|c|c|c|c|c|c|c|}
\hline \multirow[b]{2}{*}{$\begin{array}{l}\text { Filling of } \\
\text { counting } \\
\text { chamber }\end{array}$} & \multicolumn{2}{|c|}{ Suspension A } & \multicolumn{2}{|c|}{ Suspension B } & \multicolumn{2}{|c|}{ Suspension C } \\
\hline & $\begin{array}{l}\text { Mean } \\
\text { count }\end{array}$ & $\begin{array}{c}\text { Mean } \\
\text { square of } \\
\text { transformed } \\
\text { count }\end{array}$ & $\begin{array}{l}\text { Mean } \\
\text { count }\end{array}$ & $\begin{array}{c}\text { Mean } \\
\text { square of } \\
\text { transformed } \\
\text { count }\end{array}$ & $\begin{array}{l}\text { Mean } \\
\text { count }\end{array}$ & $\begin{array}{c}\text { Mean } \\
\text { square of } \\
\text { transformed } \\
\text { count }\end{array}$ \\
\hline 1 & $8 \cdot 6$ & 30 & 8 & 87 & 11 & $0 \cdot 29$ \\
\hline$a$ & & & & & & \\
\hline 3 & & & & & & \\
\hline 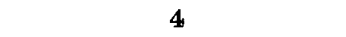 & & 0 & $\mathbf{9 \cdot 2}$ & & & \\
\hline & & & & & & \\
\hline 6 & & & & & & \\
\hline 7 & 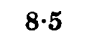 & 0.20 & 4 & $0 \cdot 22$ & 11.1 & $0 \cdot 18$ \\
\hline $\begin{array}{l}\text { Mean square attributable } \\
\text { to differences between } \\
\text { slides ( } 6 \text { D.F.) }\end{array}$ & \multicolumn{2}{|r|}{$0 \cdot 26$} & \multicolumn{2}{|r|}{0.47} & \multicolumn{2}{|r|}{$0 \cdot 17$} \\
\hline $\begin{array}{l}\text { Overall within-slide mean } \\
\text { square (203 D.F.) }\end{array}$ & \multicolumn{2}{|r|}{$0 \cdot 27$} & & & \multicolumn{2}{|r|}{0.26} \\
\hline \multicolumn{7}{|c|}{$\begin{array}{l}\text { Two analyses of the } 10 \text { a.m. samples were carried out, one for large and one } \\
\text { for small organisms, transforming to the square root of the total count on } \\
\text { each slide. For neither analysis were differences significant between slides } \\
\text { from the same sample. The mean square attributable to this source of varia- } \\
\text { bility was } 0 \cdot 21 \text { based on } 14 \text { degrees of freedom for each analysis. Thus, so far } \\
\text { as variability over a slide and differences from one slide to another were } \\
\text { concerned, the same conclusions appeared to hold for the organisms from } \\
\text { rumen contents as for the organisms in washed suspensions. } \\
\text { This experiment tested three additional sources of variability: (1) whether } \\
\text { there was a difference between top and bottom of the rumen; (2) whether } \\
\text { there were differences from one day to another; (3) whether the difference } \\
\text { between top and bottom varied from day to day. The answers obtained were } \\
\text { as follows: (1) There was no evidence of a top-bottom difference in concen- } \\
\text { tration of large organisms but the small organisms seemed to be more }\end{array}$} \\
\hline
\end{tabular}


concentrated at the top. The $8 \%$ difference between top and bottom did not quite reach significance at the $5 \%$ level. (2) There were large day-to-day differences in the concentration of both large and small organisms $(\boldsymbol{P}<0.001)$. When the mean concentrations were plotted against time of sampling, as in Fig. 2, the 3 p.m. samples from the middle of the rumen agreed well with the corresponding 10 a.m. samples, indicating, as expected, that the concentration changed gradually with time. Fig. 2 also revealed a positive day-to-day correlation between the concentrations of large and small organisms. (3) The difference between top and bottom did not vary significantly from day to day.

\section{DISCUSSION}

From the results of the experiments described above, it would seem that the MacMaster type of counting chamber used with suspensions of ciliates or samples of rumen contents prepared by the methods described, will enable a count of the organisms to be made with an accuracy which can be determined, and which varies according to the numbers counted. A further implication of the results of Expt. $a$ and $b$ is that the method developed for sampling and mixing does not appear to introduce any additional source of error. It would thus not be necessary to take a large number of samples from a suspension for counting. For any given concentration the total number of fields counted is the important factor which decides the accuracy of an estimate, and three slides should be ample as a check against an error in filling a slide. From the result of Expt. $c$, namely that the position-by-day interaction was not significant, it seemed likely that repeat samples from a given position in the rumen would not vary more than might be expected on the basis of a random distribution of protozoa in that neighbourhood. In order to examine differences in concentration over the rumen, the top and bottom were chosen, as they seemed most likely to represent the extremes of protozoan concentration, as one might expect a higher concentration of organisms to be associated with the greater quantity of hay fragments nearer the top of the rumen. Although there is danger in generalizing, in our case it appeared that the difference in concentration might not be more than about $10 \%$ in favour of the top. Should this be the case generally, then it can be shown that provided no more than 200 organisms/slide are counted, the standard deviation will be only about $10 \%$ greater than if the distribution were random over the rumen. It has also been clearly demonstrated that the concentration of organisms changed with time, and that both large and small organisms followed a similar trend. From the magnitude of these changes and from such information as exists in the literature concerning differences between sheep (Williams \& Moir, 1951), there is little doubt that variability arising from those two sources would swamp such variability as exists in the sampling methods described in this paper.

The authors wish to express their indebtedness to Dr A. E. Oxford, for much helpful discussion.

A large part of this work was incorporated by one of us (J.M.E.) in a thesis submitted (1956) to the University of Aberdeen for a Degree of Ph.D. 


\section{REFERENCES}

Adam, K. M. G. (1951). The quantity and distribution of the ciliate protozoa in the large intestine of the horse. Parasitology, 41, 301.

Adam, K. M. G. (1953). In vivo observations on the ciliate protozoa inhabiting the large intestine of the horse. J. gen. Microbiol. 9, 376.

Anscombe, F. J. (1948). The transformation of Poisson, binomial and negativebinomial data. Biometrika, 35, 246.

Ferber, K. E. (1928). Die Zahl und Masse der Infusorien im Pansen und ihre Bedeutung für den Eiweissaufbau der Wiederkäuer. Z. Tierz. ZüchtBiol. 12, 31.

Ferber, K. E. \& Winogradawa-Federowa, T. (1929). Zählung und Teilungsquote der Infusorien im Pansen der Wiederkäuer. Biol. Zbl. 49 (6), 321.

Gil_BerT, J. Y. (1942). The errors of the Sedgwick-Rafter counting chamber in the enumeration of Phytoplankton. Trans. Amer. micr. Soc. 61, 217.

GutierRez, J. (1955). Experiments on the culture and physiology of holotrichs from the bovine rumen. Biochem. J. 60, 516.

Hall, R. P., Johnson, D. F. \& Loefer, J. B. (1935). A method for counting protozoa in the measurement of growth under experimental conditions. Trans. Amer. micr. Soc. 54, 298.

Heald, P. J., Oxford, A. E. \& Sugden, B. (1952). A convenient method for preparing massive suspensions of virtually bacteria-free ciliate protozoa of the genera Isotricha and Dasytricha for monometric studies. Nature, Lond. 169, 1055.

Hungate, R. E. (1942). The culture of Eudiplodinium neglectum, with experiments on the digestion of cellulose. Biol. Bull., Woods Hole, 83, 303.

Johnson, B. C., Hamilton, R. S., Robinson, W. R. \& Garey, J. C. (1944)). On the mechanism of non-protein-nitrogen utilization by ruminants. J. Anim. Sci. 3, 287.

Mangold, E. (1929). Handbuch der Ernährung und des Stoffwechsels der Landwirtschaftlichen Nutztiere, Band II. Berlin: Julius Springer.

Morr, R. J. (1951). The seasonal variation in the ruminal micro-organisms of grazing sheep. Aust. J. agric. Res. 2, 322.

Morr, R. J. \& Somers, M. (1956). A factor influencing the protozoal population in Sheep. Nature, Lond. 178, 1472.

Mowry, H. A. \& Becker, E. R. (1930). Experiments on the biology of infusoria inhabiting the rumen of goats. Iowa St. Coll. J. Sci. 5, 35.

OxFord, A. E. (1955). The rumen protozoa: their chemical composition, metabolism, requirements for maintenance and culture and physiological significance for the host. Exp. Parasitol. 4, 569.

Quenouille, M. H. (1950). Introductory Statistics. London: Butterworth-Springer.

'STUdent' (1907). On the error of counting with a haemocytometer. Biometrika, 5, 351.

Wath, J. G. VAN Der \& Myburgh, S. (1941). Studies on the alimentary tract of merino sheep in South Africa. VI. The role of infusoria in ruminant digestion with some remarks on ruminant bacteria. Onderstepoort J. vet. Sci. 17, 61 .

Weller, R. A. \& GraY, F. V. (1954)). The passage of starch through the stomach of the sheep. J. exp. Biol. 31, 40.

Williams, V. J. \& MoIr, R. J. (1951). Ruminal Flora Studies in the sheep. III. The influence of different sources of nitrogen upon nitrogen retention and upon the total number of free micro-organisms in the rumen. Aust. J. sci. Res. 4B, 377. 